\title{
Dephasing of excitons and multiexcitons in undoped and $p$-doped InAs/GaAs quantum dots-in-a-well
}

\author{
Valentina Cesari and Wolfgang Langbein* \\ Cardiff University School of Physics and Astronomy, The Parade, Cardiff CF24 3AA, United Kingdom \\ Paola Borri \\ Cardiff University School of Biosciences, Museum Avenue, Cardiff CF10 3AX, United Kingdom
}

(Received 10 June 2010; published 11 November 2010)

\begin{abstract}
We report an experimental investigation of the dephasing of excitons and multiexcitons in technologically relevant undoped and $p$-doped InAs/GaAs dot-in-a-well structures emitting near $1.3 \mu \mathrm{m}$ wavelength. Using a transient four-wave mixing technique in heterodyne detection, we measured the excitonic dephasing due to phonon coupling in the temperature range from 5 to $300 \mathrm{~K}$, and the multiexcitonic dephasing at low temperature by electrically injecting carriers through a $p-i-n$ diode structure. While the temperature-dependent excitonic dephasing is found to be similar to previous studies, the contribution from electrically injected carriers is weaker in these dot-in-a-well systems due to a reduced pure dephasing from Coulomb interaction with carriers in the barrier material. Moreover, multiexcitonic transitions contribute with a subpicosecond dephasing, corresponding to a homogeneous broadening in the $\mathrm{meV}$ range. In the $p$-doped structure, positively charged multiexcitons are formed due to the built-in hole reservoir, which show a dominating dephasing component in the subpicosecond range. However, a weaker component in the $10 \mathrm{ps}$ range is observed and attributed to final states with spin-forbidden relaxation.
\end{abstract}

DOI: 10.1103/PhysRevB.82.195314

PACS number(s): 78.47.nj, 42.50.Md, 78.67.Hc

\section{INTRODUCTION}

The optical properties of semiconductor quantum dots (QDs) have been the subject of intense experimental and theoretical study in the last decade. This effort was driven, besides fundamental interest of quantum phenomena in solid-state matter, by the wide range of possible applications of these nanostructures. Epitaxially grown QDs embedded in a crystalline environment are easily integrated in semiconductor devices for optoelectronic applications. Among these, InGaAs/GaAs QDs are the most studied material system. Their emission in the optical communication wavelength window, combined with expected superior device performances arising from the reduced dimensionality, has triggered large fabrication and characterization efforts. ${ }^{1}$ One example is the introduction of a dot-in-a-well (DWELL) structure, where InAs QDs are embedded in an InGaAs quantum well sandwiched between GaAs barriers, which has enabled the fabrication of QD lasers with high efficiency of carrier capture into the QDs and emission near $1.3 \mu \mathrm{m}$ wavelength. ${ }^{2}$

Besides optoelectronics, coherent light-matter interaction in QDs is also receiving attention for solid-state implementations of quantum information processing. ${ }^{3,4}$ Phenomena such as Rabi rotations, cavity quantum electrodynamics in the weak- and strong-coupling regime, ultralow threshold lasers, and single photon sources providing indistinguishable and/or polarization-entangled photons have been recently demonstrated, using InGaAs/GaAs QDs as two-level systems with large transition dipole moments compared to atoms and yet narrow homogeneous linewidths compared to semiconductor structures of higher dimensionality.

The dephasing of an excitonic transition in a QD is of crucial importance for all these applications. The dephasing time sets the time scale during which the coherence is preserved and therefore operations based on coherent lightmatter interaction can be performed. ${ }^{5}$ The homogeneous broadening, given by the inverse dephasing time, is a key parameter for the coupling regimes of QDs in nanocavities, and the performance of low threshold nanolasers. ${ }^{3}$ Recently, the role of dephasing has been the subject of intense debates, both theoretically ${ }^{6-8}$ and experimentally, ${ }^{9-11}$ to explain the "cavity-feeding" effect, a significant cavity mode emission even for large spectral detuning from the excitonic resonance of a single QD emitter. The homogeneous broadening has also an important influence on the optical gain of QD lasers and amplifiers. ${ }^{12}$ The dephasing is determined by intrinsic dynamics such as radiative processes, carrier-phonon scattering and carrier-carrier scattering. Hence its study is a direct probe of these fundamental processes and also of the dynamics which ultimately limits the speed of QD-based optoelectronics devices. ${ }^{13}$

Despite its importance, the measurement of the exciton dephasing time in InGaAs/GaAs quantum dots has proven to be challenging due to spectral jitter hampering simple linewidth measurements. Using a nonlinear optical technique, four-wave mixing, these effects were eliminated, ${ }^{14-16}$ revealing dephasing times of the ground-state (GS) excitonic transition in the nanosecond range at low temperatures $(\sim 5 \mathrm{~K})$, decreasing to subpicosecond values at room temperature, and corresponding to homogeneous linewidths ranging over 4 orders of magnitude from $1 \mu \mathrm{eV}$ to $10 \mathrm{meV}$. Moreover, an unusual nonexponential dephasing was observed, corresponding to a non-Lorentzian homogeneous line shape with a narrow Lorentzian zero-phonon line (ZPL) superimposed on a broad acoustic-phonon band which stimulated theoretical works on the exciton-phonon coupling in QDs. ${ }^{17,18}$ More recently, the long ZPL dephasing time at low tempera- 
ture was shown to reach its radiative limit on a series of InGaAs/GaAs QDs with different quantum confinement potentials $^{19,20}$ and on strain-compensated InAs QDs emitting around $1.5 \mu \mathrm{m}$ wavelength, reaching several nanoseconds at low temperatures. ${ }^{21}$

Beyond one-exciton transitions, multiexciton complexes are particularly relevant in systems such as nonresonantly pumped QDs coupled to nanocavities, ${ }^{22}$ and QD lasers and amplifiers which operate in the regime of population inversion. However, even less experimental work has been reported on multiexcitonic dephasing in InGaAs QDs using four-wave mixing. ${ }^{13,23}$ From single-dot photoluminescence measurements, only a lower limit for the dephasing can be gained from the transition linewidths, which show a significant broadening of higher multiexcitonic transitions. ${ }^{24,25}$

In this paper we report an experimental investigation of the dephasing of excitons and multiexcitons in technologically relevant undoped and $p$-doped InAs/GaAs DWELL structures emitting near $1.3 \mu \mathrm{m}$ wavelength, which are described in Sec. II together with the experimental setup used. The excitonic dephasing due to phonon coupling in the temperature range from 5 to $300 \mathrm{~K}$ is discussed in Sec. III, and the multiexcitonic dephasing at low temperature measured by electrically injecting carriers through a $p-i-n$ diode structure or by $p$ doping is given in Sec. IV.

\section{SAMPLES AND EXPERIMENT}

The investigated samples are $p$-type-intrinsic- $n$-type $(p-i-n)$ ridge waveguide structures of $4 \mu \mathrm{m}$ width and 0.5 $\mathrm{mm}$ length, allowing for electrical injection. They contain in the intrinsic region 10 InGaAs DWELL layers separated by $33 \mathrm{~nm}$ GaAs spacers, sandwiched between $1.5-\mu \mathrm{m}$-thick AlGaAs cladding layers. In one of the samples modulation $p$ doping was provided by a 10-nm-thick layer of carbondoped GaAs in the spacer, ending $9 \mathrm{~nm}$ below each dot-inwell layer (doping level $\sim 8$ acceptors per dot). Samples were processed with tilted facets $\left(\sim 7^{\circ}\right)$ to suppress backreflection into the waveguide mode and lasing. Roomtemperature amplified spontaneous emission spectra ${ }^{26}$ showed an inhomogeneous broadening of the GS excitonic transition in the QD ensemble of $\sim 36 \mathrm{meV}$, and emission from the first optically active excited state (ES) transition at $60 \mathrm{meV}$ above the GS. The GS emission wavelength is similar in both samples and shifts from $1.27 \mu \mathrm{m}$ at room temperature to $1.18 \mu \mathrm{m}$ at $20 \mathrm{~K}$. Modal gain measurements demonstrated the presence of built-in holes in the $p$-doped sample, which at low temperature shows no GS absorption in absence of electrical injection, ${ }^{27}$ giving evidence of complete filling of the hole GS.

The dephasing was measured using a transient degenerate four-wave mixing (FWM) experiment in heterodyne detection, similar to our previous works. ${ }^{15,28}$ Two colinearly polarized $\sim 100$ fs Fourier-limited pulses at $\nu_{\mathrm{r}}=76 \mathrm{MHz}$ repetition rate were provided by an optical parametric oscillator (APE PP2) at a wavelength tuned to the center of the GS transition and were coupled into the transverse electric waveguide mode. Their intensities were adjusted to give rise to a FWM in the third-order regime and correspond to
10-100 $\mu \mathrm{W}$ average beam power. The delay time $\tau$ between the two pulses was controlled with femtosecond resolution using a mechanical stage and an optical delay line. Pulse 1 was leading pulse 2 for positive $\tau$. In the heterodyne technique pulse 1 (2) is frequency upshifted by the radio frequency $\nu_{1}=80 \mathrm{MHz}\left(\nu_{2}=79 \mathrm{MHz}\right)$ using an acoustooptic modulator and the interference of the FWM field with an unshifted reference pulse is detected background free at the pulse repetition sideband at $2 \nu_{2}-\nu_{1}-\nu_{\mathrm{r}}=2 \mathrm{MHz}$ using a pair of balanced photodiodes and a lock-in amplifier. Also the delay time between pulse 2 and reference pulse can be varied using a mechanical stage to time resolve the FWM signal. ${ }^{15}$ Importantly, through its interference with the reference field, the FWM field amplitude, rather than its intensity, is measured, resulting in a large dynamic range of typically 4 orders of magnitude field, i.e., 8 orders of magnitude intensity. Typical FWM powers at zero delay are in the $10 \mathrm{nW}$ range, corresponding to about 1000 photons per pulse. For temperature dependent studies samples were held in a specially designed cold-finger helium cryostat which allowed light coupling with high numerical aperture into and out of the ridge waveguide. Throughout the paper, we relate angular frequencies with energies using the factor $\hbar$.

\section{TEMPERATURE DEPENDENCE: EXCITON-PHONON INTERACTIONS}

The FWM from an inhomogeneously broadened transition is a photon echo in real time. The rephasing of the individual third-order polarizations from the inhomogeneous ensemble at the echo time allows to overcome the inhomogeneous broadening and to measure the intrinsic dephasing time. This is achieved by time integrating the photon-echo amplitude (TI-FWM) and measuring its decay as a function of the delay time $\tau$ between the exciting pulses. Spectral diffusion, which is a variation in the QD transition frequency over time from slow fluctuations of the QD environment, severely limits the ability to measure the intrinsic homogeneous linewidth in single-dot photoluminescence experiments requiring long integration times. ${ }^{29}$ Conversely, it does not affect the photon echo formation since it is negligible on the time scale of the rephasing which is the short delay $\tau$ between the two pulses. Hence TI-FWM photon echo enables the measurement of the intrinsic dephasing. Similar to our previous works, ${ }^{15,20,23}$ the spectral width of the excitation pulses was smaller than the QD inhomogeneous broadening, therefore only part of the inhomogeneously broadened QD distribution is probed and the echo duration is determined by the pulse duration. The effect of exciton-phonon interaction on the dephasing of the GS excitonic transition $(0-\mathrm{X})$ was investigated by measuring the FWM dynamics over a temperature range from 5 to 300 $\mathrm{K}$, in absence of electrical injection with zero voltage $\left(U_{\mathrm{C}}\right.$ $=0)$ across the $p-i$ - $n$ structure.

In Fig. 1 the TI-FWM versus delay time $\tau$ is shown for the undoped sample. It features an initial rise during pulse overlap due to the echo formation, a fast decay due to exciton-acoustic-phonon interactions, ${ }^{15,20}$ oscillations originating from exciton-biexciton $(\mathrm{X}-\mathrm{XX})$ beats, ${ }^{15,30}$ and a long exponential decay with a time constant $T_{2} / 2$, where $T_{2}$ is the 


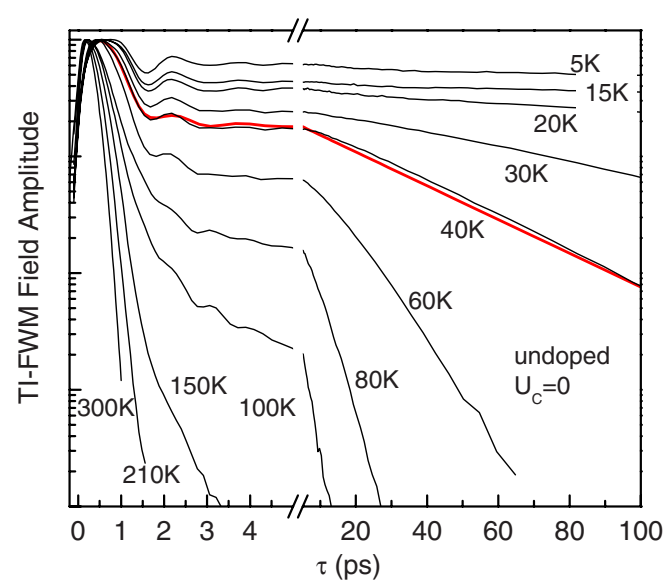

FIG. 1. (Color online) Normalized TI-FWM as function of delay on the structure in the absence of electrical injection $\left(U_{\mathrm{C}}=0\right)$ at different temperatures as indicated. The data was taken up to a maximum delay of $1 \mathrm{~ns}$. A fit (see text) to the experimental data at $40 \mathrm{~K}$ is shown (thick line).

dephasing time of the ZPL. Since during pulse overlap the TI-FWM is influenced by the nonresonant response of the GaAs barriers and cladding layers, we analyzed the TI-FWM for $\tau>0.3$ ps.

We have fitted the dynamics of the TI-FWM as

$$
\begin{aligned}
A(\tau)= & A_{0}\left(1+A_{\mathrm{ph}} e^{-\tau^{2} \sigma_{\mathrm{ph}}^{2}}\right) e^{-2 \tau / T_{2}} \\
& \times\left[2-A_{\mathrm{XX}} \cos \left(\tau E_{\mathrm{XX}}\right) e^{-\tau^{2} \sigma_{\mathrm{XX}}^{2} / 2}\right],
\end{aligned}
$$

where $A_{\mathrm{ph}}$ is the relative amplitude of acoustic-phonon band to $\mathrm{ZPL}, \sigma_{\mathrm{ph}}$ is the width of the band, inversely proportional to the acoustic-phonon-induced polarization decay time as discussed in more detail below. $E_{\mathrm{XX}}$ is the biexciton binding energy, $\sigma_{\mathrm{XX}}$ its inhomogeneous distribution in the QD ensemble, and $A_{\mathrm{XX}}$ is a weight of the exciton-biexciton transition in the ensemble FWM, given for uncharged dots by the oscillator strength ratio of $\mathrm{X}-\mathrm{XX}$ and $0-\mathrm{X}$ transition. ${ }^{30}$ The exponential decay is described by the dephasing time $T_{2}$ of the 0-X ZPL. A fit to the data at $40 \mathrm{~K}$ is shown in Fig. 1.

Let us now consider separately each part of the measured TI-FWM dynamics. Since the spectral width of the excitation pulse is larger than the biexciton binding energy, both the ground-state-exciton (0-X) transition and the excitonbiexciton $(\mathrm{X}-\mathrm{XX})$ transition in the same QD are probed. These two polarizations oscillate with different frequencies, therefore the FWM at the echo time shows their interference beat in $\tau$. In the fit function a Gaussian inhomogeneous distribution of the biexciton binding energy of variance $\sigma_{\mathrm{XX}}$ has been considered. ${ }^{31}$ From the fitted beat frequency and damping, which is not dependent on temperature, a biexciton binding energy $E_{\mathrm{XX}}=2.7 \pm 0.3 \mathrm{meV}$ and $\sigma_{\mathrm{XX}}=0.4 \pm 0.1 \mathrm{meV}$ have been inferred, similar to the values for non-DWELL structures with a comparable GS energy ${ }^{30} A_{\mathrm{XX}}$ is found to be $0.54 \pm 0.04$ for temperatures between 5 and $60 \mathrm{~K}$. At higher temperatures the superimposed fast decay due to elastic phonon interactions leads to large uncertainties in the fitted $A_{\mathrm{XX}}$ values. We therefore assumed that the biexciton parameters do not change for temperatures higher than $60 \mathrm{~K}$ and the

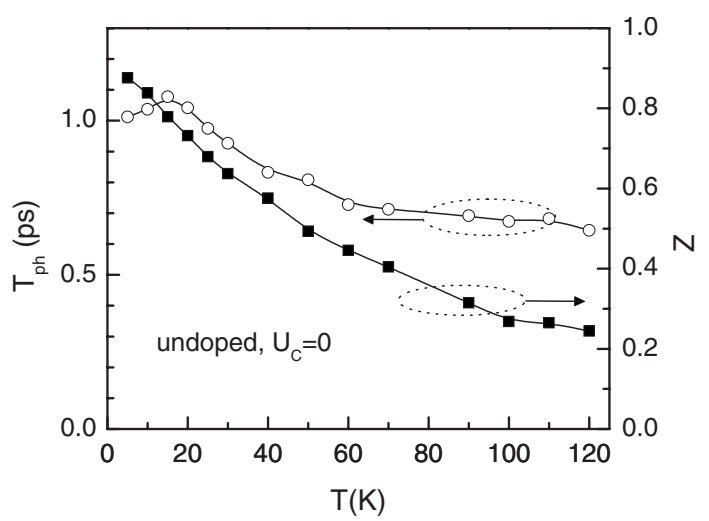

FIG. 2. Acoustic-phonon assisted dephasing time $T_{\mathrm{ph}}$ and zerophonon line weight $Z$ versus temperature for the undoped sample in absence of electrical injection.

values of $A_{\mathrm{XX}}, E_{\mathrm{XX}}$, and $\sigma_{\mathrm{XX}}$ have been kept constant. The finding of $A_{\mathrm{XX}} \neq 1$ is attributed ${ }^{30}$ to the presence of charges in the dot GS even in nominally undoped QDs since a singly charged QD only supports a charged exciton but not a charged GS biexciton due to Pauli exclusion. The charging could originate from two-photon absorption in the GaAs barrier followed by random capture into the QDs. Since thermal escape is negligible at low temperatures, the carriers remain the QDs until a carrier of opposite charge is captured by the same QD, resulting in recombination. Assuming equal weights of neutral, positively and negatively charged QDs (multiply charged QDs show GS transparency and thus no FWM), only one third of the QDs have a biexcitonic transition. However, the uncharged and charged QDs contribute equal amplitudes to the excitonic FWM signal since half of the charged QDs are spin blocked. We therefore expect $A_{\mathrm{XX}}=0.5$, in agreement with the value found in the experiment.

The initial fast decay of the TI-FWM versus $\tau$ followed by a long exponential decay corresponds in the frequency domain to a sharp Lorentzian homogeneous line superimposed to a broad band. ${ }^{15}$ This behavior is explained theoretically by the independent Boson model in terms of a narrow Lorentzian ZPL and a broadband of phonon-assisted transitions. ${ }^{20,32,33}$ The phonon band increases its weight with decreasing size of the QD exciton ${ }^{20}$ and is typically insignificant in exciton weakly confined by the lateral disorder in quantum wells. ${ }^{34,35}$ The measured acoustic-phonon assisted decay time ${ }^{17} T_{\mathrm{ph}}=\sqrt{\ln 2} / \sigma_{\mathrm{ph}}$, after which the phonon-assisted FWM [the term $\exp \left(-\tau^{2} \sigma_{\text {ph }}^{2}\right)$ in Eq. (1)] has halved, is shown in Fig. 2. $T_{\mathrm{ph}}$ shows a nonmonotonous dependence with temperature, which is due to the evolution of the acousticphonon band from an asymmetric shape on the high-energy side of the ZPL at low temperatures, for which the thermal energy is less than the typical coupled phonon energy, toward a symmetric band with increasing temperature. This leads at low temperatures to an overdamped oscillation of the acoustic-phonon-related FWM dynamics, resulting in a smaller $T_{\mathrm{ph}}$. The present results are consistent with calculations of Vagov et al. ${ }^{36}$ and extend previous experiments. ${ }^{17}$

The weight $Z$ of the ZPL in the homogeneous line shape (the fraction of the ZPL in the total absorption line shape) 


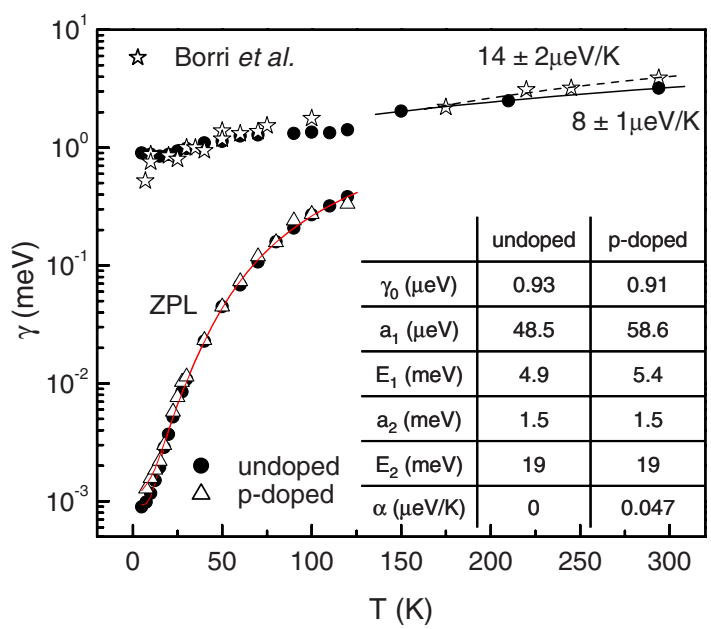

FIG. 3. (Color online) Homogeneous linewidth $\gamma$ versus temperature for the undoped (circles) and $p$-doped (triangles) sample in absence of electrical injection. Fits using Eq. (2) are shown together with the inferred parameters (within 10\% error). The linewidth in the $1 \mathrm{meV}$ range associated to the acoustic-phonon dephasing (see text) is also shown. Above $120 \mathrm{~K}$ the ZPL is no longer distinguished and a single linewidth is deduced from the Fourier transform of the TI-FWM. Data from Ref. 15, analyzed using the same procedure, are given for comparison (stars).

can be extracted from the TI-FWM from the relative contribution of the long exponential decay as described in Refs. 20 and 36. From the factor $1+A_{\mathrm{ph}} \exp \left(-\tau^{2} \sigma_{\mathrm{ph}}^{2}\right)$ in Eq. (1) we can deduce $Z^{-3}=1+A_{\mathrm{ph}}$. The resulting dependence of $Z$ on temperature is shown in Fig. 2. $Z$ decreases as the temperature increases, as expected from the independent Boson model due to the increase of the acoustic-phonon occupation number with temperature. The results in Fig. 2 are consistent with earlier experiments ${ }^{20,37}$ and calculations. ${ }^{36}$

The measured ZPL dephasing time $T_{2}$ is decreasing from $1470 \mathrm{ps}$ at $5 \mathrm{~K}$ to $3.4 \mathrm{ps}$ at $120 \mathrm{~K}$. The corresponding homogeneous broadening (full width at half maximum, FWHM) of the 0 -X transition ZPL $\gamma=2 / T_{2}$ increases with temperature, as shown in Fig. 3. The measured temperature dependence of $\gamma$ is fitted using an activated behavior ${ }^{20}$

$$
\gamma(T)=\gamma_{0}+\frac{a_{1}}{\exp \left(\frac{E_{1}}{k_{\mathrm{B}} T}\right)-1}+\frac{a_{2}}{\exp \left(\frac{E_{2}}{k_{\mathrm{B}} T}\right)-1}+\alpha T,
$$

with the Boltzmann constant $k_{\mathrm{B}}$. The zero-temperature homogeneous broadening $\gamma_{0}$ is expected to be limited by the exciton lifetime. ${ }^{19}$ To investigate this aspect we measured the exciton lifetime by differential transmission spectroscopy (DTS). DTS is implemented in our experiment by detecting at the frequency $\nu_{2}$ the change $\Delta T$ of the transmission amplitude $T_{0}$ of a weak probe pulse 2 , induced by a stronger pump pulse 1. In Fig. 4 the differential transmission in decibels $\Delta G=20 \log \left(1+\Delta T / T_{0}\right)$ is shown for the undoped and $p$-doped sample at $20 \mathrm{~K}$ in absence of electrical injection $\left(U_{\mathrm{C}}=0\right) . \Delta G$ rises with the integral of the pulse autocorrelation and decays with a time constant of $\tau_{1}=900 \mathrm{ps}$ (760 ps) for the undoped ( $p$-doped) sample. The corresponding life-

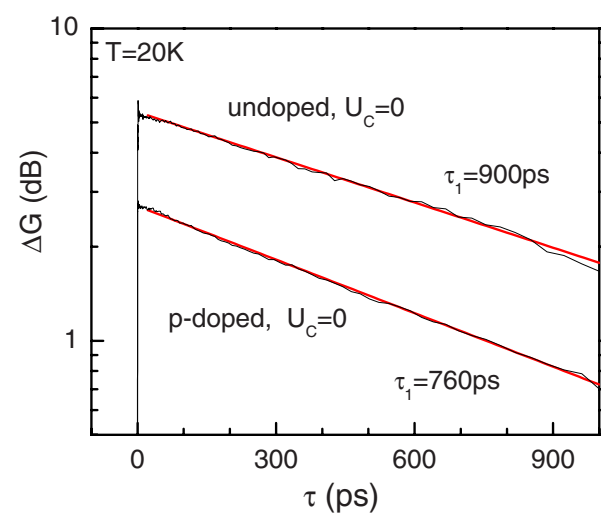

FIG. 4. (Color online) Differential transmission of the undoped and $p$-doped sample in absence of electrical injection. Thick lines are exponential fits to the data with time constants $\tau_{1}$ as given.

time limited homogeneous broadening $\gamma_{0}^{r}=1 / \tau_{1}$ of $0.73(0.86) \mu \mathrm{eV}$ is within errors $(10 \%)$ equal to $\gamma_{0}$, showing that $\gamma_{0}$ is essentially lifetime limited.

A temperature-dependent ZPL broadening is not described within the independent Boson model. In recent literature an extension of the independent Boson model accounting for pure dephasing via quadratic coupling between carriers and acoustic phonons ${ }^{18}$ was introduced to reproduce the temperature dependence of $\gamma$ measured in Ref. 15. Subsequent measurements on a series on InAs/GaAs quantum dots with variable quantum confinement showed ${ }^{20}$ a temperature-activated behavior of $\gamma$ well represented by Eq. (2) with constant activation energies $E_{1}=6 \mathrm{meV}$ and $E_{2}=28 \mathrm{meV}$ independent of the quantum confinement. Those measurements suggested that one-phonon absorption from the excitonic GS into excited states is not the underlying dephasing mechanism and supported the relevance of pure dephasing processes in the ZPL broadening. From the fit in Fig. 3 we found $E_{1}$ $=4.9 \mathrm{meV}\left(E_{1}=5.4 \mathrm{meV}\right)$ in the undoped ( $p$-doped) sample and $E_{2}=19 \mathrm{meV}$, similar to the results reported in previous experiments $^{15,20,38}$ and likely related to pure dephasing mechanisms as suggested in Ref. 18.

The linewidth of the acoustic-phonon broadband in the 1 $\mathrm{meV}$ range is also shown in Fig. 3. Its value is calculated as $2 \ln (2) / T_{\mathrm{ph}}$ which is the FWHM of the Fourier transform of the first-order polarization decay $\exp \left(-t^{2} \sigma_{\mathrm{ph}}^{2} / 4\right)$ obtained considering that the TI-FWM versus delay time $\tau$ in Eq. (1) is a probe of the real-time evolution of the first-order polarization at $t=2 \tau$ due to the photon echo. For comparison, the width of the acoustic-phonon band is shown for the data in Ref. 15 (stars in Fig. 3), analyzed using the same procedure, resulting in similar values. Above $120 \mathrm{~K}$ the ZPL is no longer resolved and an overall linewidth is deduced from the Fourier transform of the TI-FWM as a probe of the firstorder polarization decay. The corresponding homogeneous broadening in the few meV range scales linearly with temperature as shown in Fig. 3. For comparison, the homogeneous broadening from the data in Ref. 15 shows a stronger increase with temperature. The physical interpretation of this broadening is however quite complex since both optical and acoustic phonons have to be taken into account as well as pure and/or inelastic dephasing processes involving the excitonic exited states. 


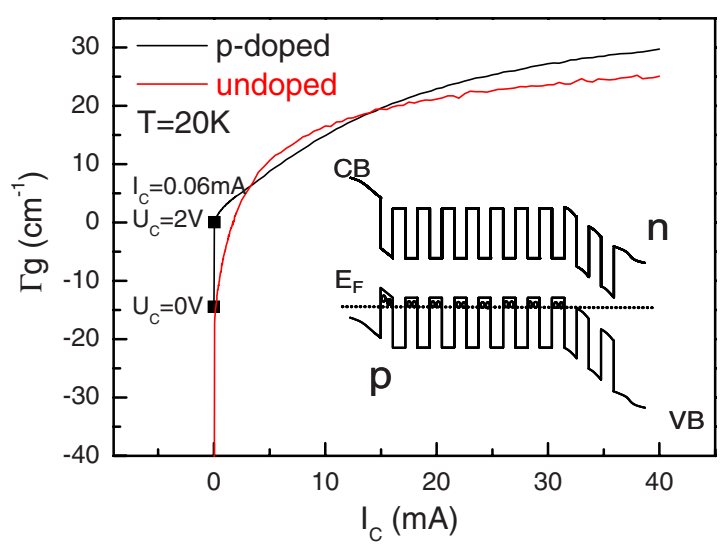

FIG. 5. (Color online) Modal gain versus injected current for the $p$-doped and undoped sample. Inset: sketch of the band configuration at zero voltage across the $p-i-n$ diode structure.

Let us comment on the dephasing measured in the $p$-doped sample in the absence of electrical injection, and the corresponding homogeneous broadening (see Fig. 3 triangles). The observation of a long dephasing appears surprising considering the presence of built-in holes which promote carrier-carrier scattering. Furthermore, in the presence of two or more holes in a QD, the GS should be transparent and no resonant FWM and DTS should be present. We attribute their observation for $U_{\mathrm{C}}=0$ to the band bending near the $n$ side, which results in a depletion of the adjacent QD layers (see sketch in Fig. 5). These depleted QDs are responsible for the FWM and DTS signal measured on the $p$-doped sample. The modal gain measured for this sample, shown in Fig. 5, confirms this interpretation. A finite absorption (negative modal gain) is observed for $U_{\mathrm{C}}=0$, while the sample is transparent (zero modal gain) at a small injection current $(0.06 \mathrm{~mA})$ and $U_{\mathrm{C}}=2 \mathrm{~V}$ for which the flat-band condition is reached, and therefore the dot GS of all layers are filled by built-in holes. The temperature dependence of $\gamma$ at $U=0 \mathrm{~V}$ of the $p$-doped sample is similar to the one of the undoped sample (see Fig. 3), supporting the attribution to depleted QDs. However, at low temperature the $p$-doped sample shows a slightly higher homogeneous broadening with respect to the undoped one, which can be described by a linear term given by the parameter $\alpha$ in Eq. (2). A homogeneous broadening linearly increasing with temperature is expected in bulk and quantum wells ${ }^{14}$ but not in QDs. ${ }^{39}$ We suggest that this contribution is due to dephasing by Coulomb scattering with the twodimensional continuum of holes present in the wetting layer of adjacent, not depleted QD layers, in which not all built-in holes are captured into the QDs due to Coulomb repulsion. The probability of these scattering processes is expected to increase linearly with temperature according to the broadening of the Fermi edge.

\section{COULOMB INTERACTIONS}

Let us now discuss the effect of Coulomb interactions on the dephasing of GS excitons, biexcitons, and multiexcitons. Pauli blocking allows no more than two carriers to occupy the twofold spin-degenerate GS of electrons $\left(e_{0}\right)$ and holes

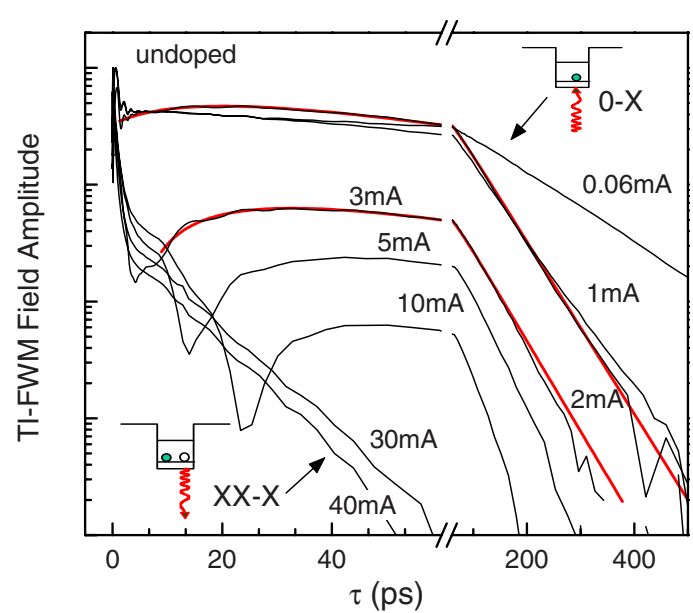

FIG. 6. (Color online) Normalized TI-FWM versus delay time for different injected currents at $20 \mathrm{~K}$ for the undoped sample. The contributions from the ground-state excitonic transition $(0-\mathrm{X})$ and the biexciton to exciton transition $(\mathrm{XX}-\mathrm{X})$ are indicated.

$\left(h_{0}\right)$ in the QD, which form the GS biexciton XX. Additional carriers have to occupy higher states. At high $(>2)$ exciton occupancies, single-dot photoluminescence spectra with a large number of emission lines of various broadenings have been reported. ${ }^{40-43}$ This is because the transition energy of the optical recombination of an electron-hole pair depends on the additional electrons and holes present in the dot being "active spectators" by Coulomb and exchange interaction. ${ }^{44,45}$ Most of the experimental work reported in the past on biexcitons and multiexcitons in InGaAs QDs focused on the energy position and strength of the optical transitions, while their homogeneous broadening, and in turn dephasing, has been less investigated.

We can progressively increase the number of excitons in the investigated samples by varying the electrical injection, and study the passage from a FWM response of empty dots, to a response of biexcitonic and multiexcitonic states. The resulting FWM dynamics of the undoped sample is shown in Fig. 6 at $20 \mathrm{~K}$ for different injection currents. Without electrical injection the $0-\mathrm{X}$ excitonic transition from the crystal ground state to GS exciton ( $e_{0} h_{0}$ pair) is probed and shows an initial fast decay followed by a long exponential decay due to the ZPL, as discussed in the previous section. At $20 \mathrm{~K}$ the ZPL dominates the line shape (see also Fig. 2). With increasing injection current the decay rate increases, showing that the ZPL broadens due to Coulomb interaction with the injected carriers.

From modal gain measurements we found a GS transparency current of $I_{\mathrm{T}}=1.7 \mathrm{~mA}$. For injection currents $I_{\mathrm{C}}<I_{\mathrm{T}}$ the probed GS transition is in the absorption regime with on average less than one $e_{0} h_{0}$ pair per QD. With increasing $I_{\mathrm{C}}$ from below to above $I_{\mathrm{T}}$, the FWM response passes from being dominated by the $0-\mathrm{X}$ to being dominated by the $\mathrm{XX}-\mathrm{X}$ transition. For a QD in the 0 state, the $0-\mathrm{X}$ transition has a population inversion of -1 while for a QD in the $\mathrm{XX}$ state the XX-X transition has a population inversion of +1 . Therefore, the optically driven polarizations of these two transitions are opposite and show a destructive interference, which is visible in Fig. 6 as a node of the TI-FWM versus 


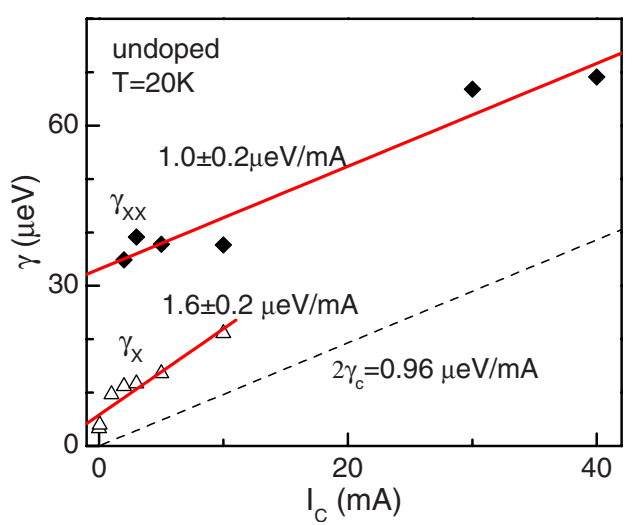

FIG. 7. (Color online) Measured homogeneous broadening FWHM of the $0-\mathrm{X}\left(\gamma_{\mathrm{X}}\right)$ and $\mathrm{XX}-\mathrm{X}\left(\gamma_{\mathrm{XX}}\right)$ transition at $20 \mathrm{~K}$ for the undoped sample. Linear fits (solid lines) versus injection current with the corresponding slopes are also shown. The estimated dephasing associated to the capture rate $\left(\gamma_{\mathrm{c}}\right)$ is shown as dashed line.

delay due to the different dephasing of the two transitions. For similar FWM amplitudes of the $0-\mathrm{X}$ and XX-X transition, which is expected close to transparency, the zero crossing occurs near zero delay. With increasing injection current the zero crossing shifts to positive delays, showing that the $0-\mathrm{X}$ transition has a longer dephasing time than the XX-X transition. The rise and decay of the FWM signal can be fitted by the difference of two exponential decays (thick line in Fig. 6). From these fits the homogeneous broadening of the $0-X\left(\gamma_{X}\right)$ and $X X-X\left(\gamma_{X X}\right)$ transitions versus injected current were deduced and are summarized in Fig. 7. An increase in both broadenings is observed with increasing $I_{\mathrm{C}}$ with a systematically higher value of $\gamma_{\mathrm{XX}}$ compared to $\gamma_{\mathrm{X}}$. Such a difference at the finite temperature of $20 \mathrm{~K}$ was found also in our earlier works ${ }^{14,23}$ and can be attributed to the larger number of phonon scattering channels for the XX-X transition which is involving three excitons.

By linearly fitting $\gamma_{\mathrm{X}}$ and $\gamma_{\mathrm{XX}}$ a slope of $\sim 1 \mu \mathrm{eV} / \mathrm{mA}$ is deduced. This value is compared with the contribution from carrier capture as follows. At low temperature a carrier which is captured into a QD does not escape and dephases the GS polarization. Thus the capture rate per dot $\gamma_{\mathrm{c}}$, which can be estimated using the equilibrium between capture and recombination rate in steady state, results in a polarization decay rate $\gamma_{\mathrm{c}}$. At transparency there is on average one exciton per dot, so that $\gamma_{\mathrm{c}}=\gamma_{\mathrm{r}} I_{\mathrm{C}} / I_{\mathrm{T}}$ using the bright exciton recombination rate $\gamma_{\mathrm{r}}=0.82 \mu \mathrm{eV}$ obtained from the decay time of about $0.8 \mathrm{~ns}$ measured by DTS. The factor of 2 reduction in the recombination rate due to the equal probability of creating a dark (spin-forbidden) or a bright (spin-allowed) exciton by electrical injection is compensated by the factor of 2 increase in the capture rate due to independent capture of electron and hole. The corresponding homogeneous broadening FWHM $2 \gamma_{\mathrm{c}}$, indicated in Fig. 7 , is comparable to the measured slope of the homogeneous broadening due to electrical injection. This finding implies that carriers coming close enough to a QD to influence their polarization are captured by this QD during this interaction. In a previous similar study on non-DWELL structures of somewhat less

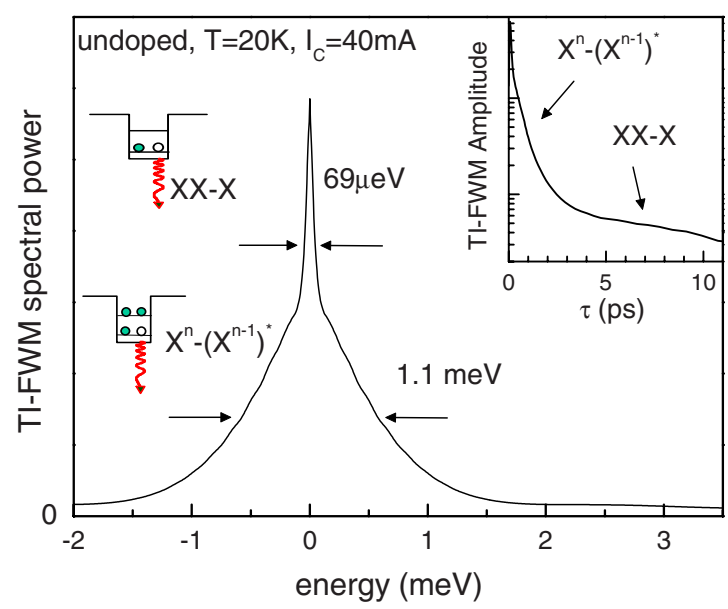

FIG. 8. (Color online) Power spectrum of the TI-FWM in the undoped sample at $20 \mathrm{~K}$ and $40 \mathrm{~mA}$ injection current. The homogeneous broadening FWHM of the biexciton to exciton transition $\mathrm{XX}-\mathrm{X}$ and of the multiexcitonic transition $\mathrm{X}^{n}-\left(\mathrm{X}^{n-1}\right)^{*}$ is indicated. In the inset the TI-FWM versus delay time is shown with an initial fast decay associated to the multiexcitonic transition and a longer dephasing of the XX-X transition.

confinement ${ }^{23}$ the capture-induced broadening was found to be an order of magnitude smaller than the current-induced broadening, indicating that injected carriers in the barrier and wetting layer regions interacting with the excitons in the QDs had only about $10 \%$ probability being captured during this interaction. The increased carrier capture efficiency in DWELL structures indicated by these results is in agreement with the studies of optical performance of DWELL quantumdot laser structures. ${ }^{2}$

For currents higher than $10 \mathrm{~mA}$, the majority of dots is populated by biexcitons or multiexcitons $\left(\mathrm{X}^{n}, n>2\right)$, with at least one carrier in the ES. The multiexcitons are mostly in their ground states due to the low thermal energy of $1.7 \mathrm{meV}$ at $20 \mathrm{~K}$ compared to the electron- and hole-level splittings. In the corresponding TI-FWM a nonexponential decay is observed, showing a fast component which is attributed to the multiexcitonic states. The related transition resonant to the GS goes from a ground-state multiexciton $\mathrm{X}^{n}$ to an excited multiexciton $\left(\mathrm{X}^{n-1}\right)^{*}$ with only one electron-hole pair in the GS and additional carriers in the excited states. This transition has a strong final state damping due to the relaxation of $\left(\mathrm{X}^{n-1}\right)^{*}$ into its ground state with two electron-hole pairs in the GS, resulting in a fast TI-FWM decay. This interpretation is consistent with direct measurements of the subpicosecond relaxation of the excited multiexciton $\left(\mathrm{X}^{n-1}\right)^{*}$ using DTS. ${ }^{27}$ The XX-X transition instead involves both initial and final states in their ground state hence has a weaker final-state damping.

The homogeneous broadening of the $\mathrm{X}^{n}-\left(\mathrm{X}^{n-1}\right)^{*}$ transitions is evaluated in Fig. 8 from the Fourier transform of the TI-FWM at high injection current $(40 \mathrm{~mA})$. The nonexponential decay, with a fast and a slow component, is shown in the inset of Fig. 8 and corresponds to a homogeneous line shape well described by the sum of two Lorentzians with linewidths as indicated. The smaller linewidth is due to the $\mathrm{XX}-\mathrm{X}$ transition while the larger linewidth in the $\mathrm{meV}$ range 


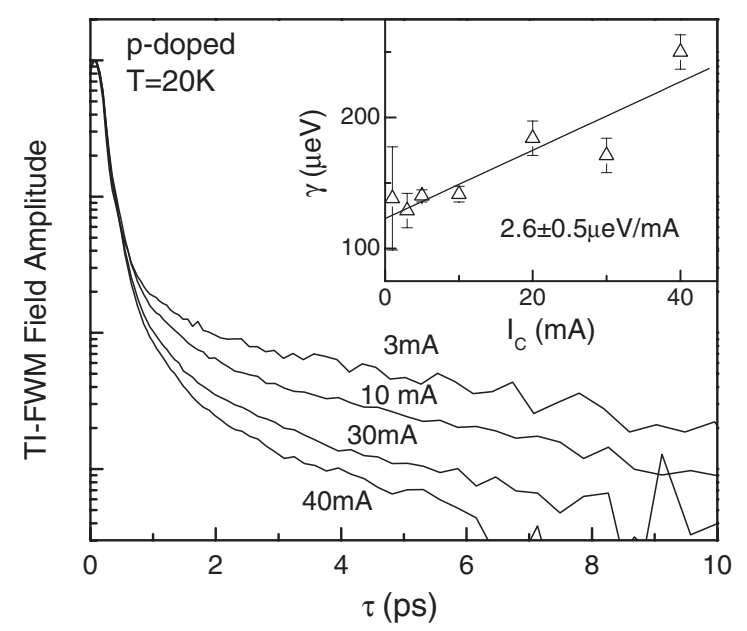

FIG. 9. Normalized TI-FWM versus delay time for different injection currents at $20 \mathrm{~K}$ for the $p$-doped sample. In the inset, the homogeneous broadening FWHM associated with the longer decay is shown together with a linear fit (solid line) as a function of injection current.

is attributed to the multiexcitonic transitions which has a large final-state damping. This linewidth increases with injection current from $0.85 \mathrm{meV}$ at $20 \mathrm{~mA}$ to $1.1 \mathrm{meV}$ at 40 $\mathrm{mA}$, which is due to the increase in the average number of excitons $n$, resulting in more carriers contributing in the relaxation process. Note that such multiexciton complexes are particularly relevant in systems such as nonresonantly pumped QDs coupled to nanocavities, ${ }^{22}$ and in QD lasers and amplifiers which operate in the regime of population inversion. The presence of a subpicosecond dephasing of multiexcitonic transitions even at low temperature, corresponding to a homogeneous broadening in the meV range, affects the performances of these devices and is among the reasons for the significant cavity mode emission observed in QDnanocavity systems at large spectral detunings.

Let us now consider the $p$-doped sample at $I_{\mathrm{C}}>0$, for which the hole GS is fully occupied, while the electron GS is occupied by an average electron number proportional to $I_{\mathrm{C}}$. At very small currents $I_{\mathrm{C}}=0.06 \mathrm{~mA}$ only instantaneous FWM is observed, which is attributed to nonresonant response of the barrier and cladding layers while the QD GS transition is Pauli blocked due to occupation of the two $h_{0}$ states by built-in holes. For higher currents, a significant fraction of dots contain injected electrons and population inversion of the GS transition, resulting in optical gain proportional to this fraction is observed (see Fig. 5). The resulting TI-FWM is given in Fig. 9, showing a dominating subpicosecond decay followed by a slower exponential component on a $10 \mathrm{ps}$ time scale. A subpicosecond dephasing is expected due to the presence of built-in holes in the ES, yielding a strong final-state damping of the probed GS transition where the final state is an excited multihole state with one $h_{0}$ less (see sketch in Fig. 10), similar to the case of the $\mathrm{X}^{n}$ $-\left(\mathrm{X}^{(n-1)}\right)^{*}$ transition. The observed slower component hints to a fraction of the transitions which have a slower relaxation of the final state. We attribute this observation to a spinforbidden relaxation.
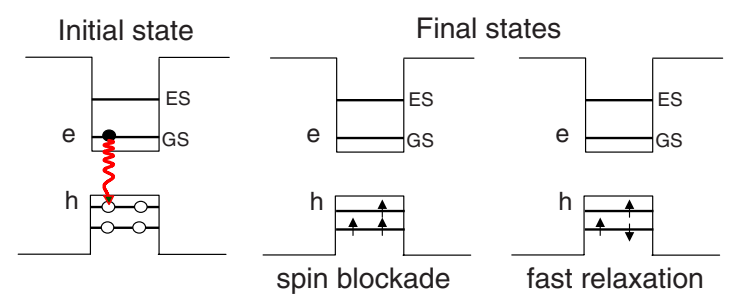

FIG. 10. (Color online) Sketch of the optical transition and subsequent damping of the three-hole final state in the $p$-doped sample.

Due to the hole Coulomb energy of about $25 \mathrm{meV}$ in the QDs, ${ }^{46}$ one can estimate from the hole confinement of about $100 \mathrm{meV}$ that even in the presence of $p$ doping providing eight holes per QD, only $\sim 4$ holes occupy the QD, with the remaining holes staying in the wetting layer. Hence for $I_{\mathrm{C}}$ $>0.06 \mathrm{~mA}$ the probed transition in the FWM experiment is as sketched in Fig. 9. The final-state damping is then due to the relaxation of the excited three-hole state. This relaxation is ultrafast, on the $100 \mathrm{fs}$ time scale, mediated by carriercarrier and carrier-phonon scattering, unless the hole spins are aligned in which case relaxation requires a spin-flip process. The probability that two holes in the excited states have the same spin orientation as the empty $h_{0}$ state is $1 / 6$, considering equal probabilities of the possible spin $(\uparrow, \downarrow)$ and spatial orbital $\left(p_{x}, p_{y}\right)$ configurations: $\left(p_{x} \uparrow, p_{y} \uparrow\right),\left(p_{x} \downarrow, p_{y} \downarrow\right)$, $\left(p_{x} \uparrow, p_{x} \downarrow\right), \quad\left(p_{y} \uparrow, p_{y} \downarrow\right), \quad\left(p_{x} \downarrow, p_{y} \uparrow\right), \quad$ and $\quad\left(p_{x} \uparrow, p_{y} \downarrow\right)$. The slower component could thus be due to dots occupied by two excited holes with parallel spin, which is also parallel to the spin of the GS hole removed by the optical transition. Note that if only three holes occupy the QD, the probability that the hole in the ES has a spin parallel to the hole left in the GS is $1 / 2$. Vice versa, if five holes or more occupy the QD, one hole in the ES will always have spin antiparallel to the hole left in the GS and no spin blockade will occur. The observed ratio of about a factor 50 between fast and slow components would in this scenario be explained by an ensemble of dots occupied by four or five holes per dot.

Single quantum-dot photoluminescence measurements in less confined dots with a maximum charging of three electrons showed such spin-blockade behavior on the linewidth for the triply negatively charged exciton ${ }^{47}$ which exhibited a fast dephasing for the singlet state (4 meV linewidth), and longer dephasing ( $0.4 \mathrm{meV}$ linewidth) for the energetically higher triplet state, which above $10 \mathrm{~K}$ is thermally excited. Similar experiments ${ }^{48}$ on InAs QDs emitting at the same wavelength as our samples, but not DWELL structures, i.e., having larger confinement, showed charging sequences up to six holes, with emission lines showing broadenings of $0.1-1$ $\mathrm{meV}$ at $4.5 \mathrm{~K}$. Furthermore, a significant temperaturedependent broadening was observed for multiply negatively charge excitons, ${ }^{49}$ leading to broadenings up to $1 \mathrm{meV}$ at 20 $\mathrm{K}$. These reports are generally consistent with the proposed spin-blockade mechanism of the slower dephasing but a direct comparison with single quantum-dot emission spectra from the same QD type is not available.

The slower component has a dephasing time which decreases with increasing injection current. The corresponding homogeneous broadening is shown in the inset of Fig. 8. It shows a slope significantly higher than the one measured for 
the $0-\mathrm{X}$ and $\mathrm{XX}-\mathrm{X}$ dephasing which was attributed to carrier capture (see Fig. 7). To estimate the carrier injection rate in the $p$-doped device, which has a nominally equal geometry, we assume that its dot density is equal such that modal gain for empty dots would be $\Gamma g=-45 / \mathrm{cm}$ as measured on the undoped sample. Using the slope of $\Gamma g$ versus $I_{\mathrm{C}}$ of $1.5 /(\mathrm{mA} \mathrm{cm})$ (see Fig. 5) and the measured carrier lifetime of $300 \mathrm{ps}$ in the $p$-doped sample at $20 \mathrm{~K}$ and $\Gamma g=15 / \mathrm{cm}$, the recombination is resulting in a carrier capture induced broadening of only $0.3 \mu \mathrm{eV} / \mathrm{mA}$, an order of magnitude below the measured broadening. We thus see a significantly stronger carrier-induced dephasing in the $p$-doped sample, which we attribute to the Coulomb interaction between the resident holes in the QDs and the injected carriers.

\section{SUMMARY}

We have performed an experimental study of the dephasing time of the ground-state excitonic transition in technologically relevant undoped and $p$-doped InAs/GaAs dots-inwell structures emitting near $1.3 \mu \mathrm{m}$. The dephasing time is measured to be $1.5 \mathrm{~ns}$ at $5 \mathrm{~K}$, and is dominated by the exciton lifetime. With increasing temperature the dephasing exhibits a strongly nonexponential behavior corresponding to a non-Lorentzian homogeneous line shape with a sharp zerophonon line superimposed onto a broad acoustic-phonon band. The dynamics is fitted with a single expression from which all relevant parameters (ZPL dephasing, acousticphonon broadening, biexciton binding energy, and distribution) are inferred. Furthermore, at low temperature in the presence of electrically injected carriers, an additional dephasing due to Coulomb interactions is observed. Differently from previous studies, the contribution from carrier capture is found to fully account for the dephasing of the excitonic and biexcitonic transitions in undoped structures, consistent with the improved capture efficiency expected for dots-in-well structures. Multiexcitonic transitions contribute with a subpicosecond dephasing, corresponding to a homogeneous broadening in the meV range, relevant for the significant cavity mode emission of nonresonantly pumped QDs coupled to nanocavities for large spectral detuning. In the $p$-doped structure, the built-in holes result in a subpicosecond dephasing, but also a slow component in the 10 ps range is observed and attributed to spin blockade of the relaxation of the multihole final state.

\section{ACKNOWLEDGMENTS}

Part of this work was supported by the Engineering and Physical Sciences Research Council (EPSRC) under Grant No. EP/E056385. The devices were processed by M. Rossetti and A. Fiore at EPF Lausanne, Switzerland and grown by S. Mikhrin, I. Krestnikov, and A. Kovsh at Innolume $\mathrm{GmbH}$, Dortmund, Germany. *langbeinww@cf.ac.uk

${ }^{1}$ D. Bimberg, M. Grundmann, and N. N. Ledentsov, Quantum Dot Heterostructures (Wiley, Chichester, 1999).

${ }^{2}$ G. T. Liu, A. Stintz, H. Li, T. C. Newell, A. L. Gray, P. M. Varangis, K. J. Malloy, and L. F. Lester, IEEE J. Quantum Electron. 36, 1272 (2000).

${ }^{3}$ P. Michler, in Single Quantum Dots: Fundamentals, Applications, and New Concepts, Topics in Applied Physics Vol. 90, edited by P. Michler (Springer, Berlin, Germany, 2003), pp. 315-47.

${ }^{4}$ Semiconductor Quantum Bits, edited by F. Henneberger and O. Benson (Pan Stanford, Singapore, 2009).

${ }^{5}$ A. Zrenner, E. Beham, S. Stufler, F. Findeis, M. Bichler, and G. Abstreiter, Nature (London) 418, 612 (2002).

${ }^{6}$ A. Naesby, T. Suhr, P. T. Kristensen, and J. Mørk, Phys. Rev. A 78, 045802 (2008).

${ }^{7}$ M. Yamaguchi, T. Asano, and S. Noda, Opt. Express 16, 18067 (2008).

${ }^{8}$ A. Auffèves, J.-M. Gérard, and J.-P. Poizat, Phys. Rev. A 79, 053838 (2009).

${ }^{9}$ J. Suffczyński, A. Dousse, K. Gauthron, A. Lemaître, I. Sagnes, L. Lanco, J. Bloch, P. Voisin, and P. Senellart, Phys. Rev. Lett. 103, 027401 (2009).

${ }^{10}$ S. Ates, S. M. Ulrich, A. Ulhaq, S. Reitzenstein, A. Löffler, S. Höfling, A. Forchel, and P. Michler, Nat. Photonics 3, 724 (2009).

${ }^{11}$ D. Englund, A. Majumdar, A. Faraon, M. Toishi, N. Stoltz, P. Petroff, and J. Vuckovic, Phys. Rev. Lett. 104, 073904
(2010).

${ }^{12}$ Peter Blood, IEEE J. Sel. Top. Quantum Electron. 15, 808 (2009).

${ }^{13}$ P. Borri, W. Langbein, S. Schneider, U. Woggon, R. L. Sellin, D. Ouyang, and D. Bimberg, IEEE J. Sel. Top. Quantum Electron. 8, 984 (2002).

${ }^{14}$ P. Borri, W. Langbein, J. M. Hvam, and F. Martelli, Phys. Rev. B 60, 4505 (1999).

${ }^{15}$ P. Borri, W. Langbein, S. Schneider, U. Woggon, R. L. Sellin, D. Ouyang, and D. Bimberg, Phys. Rev. Lett. 87, 157401 (2001)

${ }^{16}$ D. Birkedal, K. Leosson, and J. M. Hvam, Phys. Rev. Lett. 87, 227401 (2001).

${ }^{17}$ A. Vagov, V. M. Axt, T. Kuhn, W. Langbein, P. Borri, and U. Woggon, Phys. Rev. B 70, 201305(R) (2004).

${ }^{18}$ E. A. Muljarov and R. Zimmermann, Phys. Rev. Lett. 93, 237401 (2004).

${ }^{19}$ W. Langbein, P. Borri, U. Woggon, V. Stavarache, D. Reuter, and A. D. Wieck, Phys. Rev. B 70, 033301 (2004).

${ }^{20}$ P. Borri, W. Langbein, U. Woggon, V. Stavarache, D. Reuter, and A. D. Wieck, Phys. Rev. B 71, 115328 (2005).

${ }^{21}$ J. Ishi-Hayase, K. Akahane, N. Yamamoto, M. Sasaki, M. Kujiraoka, and K. Ema, Appl. Phys. Lett. 91, 103111 (2007).

${ }^{22}$ M. Winger, T. Volz, G. Tarel, S. Portolan, A. Badolato, K. J. Hennessy, E. L. Hu, A. Beveratos, J. Finley, V. Savona, and A. Imamoglu, Phys. Rev. Lett. 103, 207403 (2009).

${ }^{23}$ P. Borri, W. Langbein, S. Schneider, U. Woggon, R. L. Sellin, D. Ouyang, and D. Bimberg, Phys. Rev. Lett. 89, 187401 
(2002).

${ }^{24}$ J. J. Finley, A. D. Ashmore, A. Lemaître, D. J. Mowbray, M. S. Skolnick, I. E. Itskevich, P. A. Maksym, M. Hopkinson, and T. F. Krauss, Phys. Rev. B 63, 073307 (2001).

${ }^{25}$ B. Urbaszek, R. J. Warburton, K. Karrai, B. D. Gerardot, P. M. Petroff, and J. M. Garcia, Phys. Rev. Lett. 90, 247403 (2003).

${ }^{26}$ V. Cesari, W. Langbein, P. Borri, M. Rossetti, A. Fiore, S. Mikhrin, I. Krestnikov, and A. Kovsh, Appl. Phys. Lett. 90, 201103 (2007).

${ }^{27}$ V. Cesari, W. Langbein, and P. Borri, Appl. Phys. Lett. 94, 041110 (2009).

${ }^{28}$ P. Borri, W. Langbein, J. Mørk, and J. M. Hvam, Opt. Commun. 169, 317 (1999).

${ }^{29}$ B. Patton, W. Langbein, and U. Woggon, Phys. Rev. B 68, 125316 (2003).

${ }^{30}$ W. Langbein, P. Borri, U. Woggon, V. Stavarache, D. Reuter, and A. D. Wieck, Phys. Rev. B 69, 161301(R) (2004).

${ }^{31}$ W. Langbein, J. M. Hvam, M. Umlauff, H. Kalt, B. Jobst, and D. Hommel, Phys. Rev. B 55, R7383 (1997).

${ }^{32}$ B. Krummheuer, V. M. Axt, and T. Kuhn, Phys. Rev. B 65, 195313 (2002).

${ }^{33}$ A. Vagov, V. M. Axt, and T. Kuhn, Phys. Rev. B 66, 165312 (2002).

${ }^{34}$ E. Peter, J. Hours, P. Senellart, A. Vasanelli, A. Cavanna, J. Bloch, and J. M. Gérard, Phys. Rev. B 69, 041307(R) (2004).

${ }^{35}$ G. Mannarini and R. Zimmermann, Phys. Rev. B 73, 115325 (2006).

${ }^{36}$ A. Vagov, V. M. Axt, and T. Kuhn, Phys. Rev. B 67, 115338 (2003).

${ }^{37}$ P. Borri and W. Langbein, J. Phys.: Condens. Matter 19, 295201
(2007).

${ }^{38}$ P. Borri, S. Schneider, W. Langbein, and D. Bimberg, J. Opt. A, Pure Appl. Opt. 8, S33 (2006).

${ }^{39}$ D. Gammon, E. S. Snow, B. V. Shanabrook, D. S. Katzer, and D. Park, Science 273, 87 (1996).

${ }^{40}$ E. Dekel, D. Gershoni, E. Ehrenfreund, D. Spektor, J. M. Garcia, and P. M. Petroff, Phys. Rev. Lett. 80, 4991 (1998).

${ }^{41}$ A. Zrenner, J. Chem. Phys. 112, 7790 (2000).

${ }^{42}$ A. Hartmann, Y. Ducommun, E. Kapon, U. Hohenester, and E. Molinari, Phys. Rev. Lett. 84, 5648 (2000).

${ }^{43}$ E. Dekel, D. Regelman, D. Gershoni, E. Ehrenfreund, W. V. Schoenfeld, and P. M. Petroff, Phys. Rev. B 62, 11038 (2000).

${ }^{44}$ F. Troiani, U. Hohenester, and E. Molinari, Phys. Rev. B 62, R2263 (2000).

${ }^{45}$ A. J. Williamson, A. Franceschetti, and A. Zunger, Europhys. Lett. 53, 59 (2001).

${ }^{46}$ D. Reuter, P. Kailuweit, A. D. Wieck, U. Zeitler, O. Wibbelhoff, C. Meier, A. Lorke, and J. C. Maan, Phys. Rev. Lett. 94, 026808 (2005).

${ }^{47}$ B. Urbaszek, R. J. Warburton, K. Karrai, C. Schulhauser, A. Hogele, E. J. McGhee, A. O. Govorov, B. D. Gerardot, X. Marie, T. Amand, and P. M. Petroff, Acta Phys. Pol. A 106, 395 (2004).

${ }^{48}$ M. Ediger, K. Karrai, A. Badolato, P. M. Petroff, and R. J. Warburton, Phys. Status Solidi C 3, 3806 (2006).

${ }^{49}$ B. Urbaszek, E. J. McGhee, M. Krüger, R. J. Warburton, K. Karrai, T. Amand, B. D. Gerardot, P. M. Petroff, and J. M. Garcia, Phys. Rev. B 69, 035304 (2004). 